\title{
Revista de Relaciones Internacionales, Estrategia y Seguridad

\section{Corrupción, integración y regionalismo en las relaciones internacionales: una discusión de los sustentos de su necesidad causal*}

\author{
Diego Jiménez Cabrera ${ }^{a}$
}

\begin{abstract}
Resumen: Pocas dudas existen acerca de que la corrupción es el nodo final de una larga cadena de entidades que están vinculadas causalmente y, a la vez, es un fenómeno que puede perturbar de forma severa los procesos de integración regional, así como de regionalización. Esta creencia, altamente arraigada en la comunidad científica que estudia aquel fenómeno, ha dado lugar a que se publiquen numerosos estudios que no combinan métodos de investigación ni enfoques epistemológicos que diluciden modelos causales robustos. Así, el presente artículo de revisión pretende someter a discusión los fundamentos de la necesidad causal subyacente a los estudios en relaciones internacionales de la corrupción en su relación con la integración y el regionalismo. Como principal conclusión, se señala la necesidad de un eclecticismo metodológico y epistemológico que permita explicar las relaciones causales como una posibilidad de ampliar el análisis de causalidad, a partir de conceptos que cuenten con unidades observables y no observables desde un mayor pluralismo metodológico.
\end{abstract}

Palabras clave: causalidad; corrupción; integración; regionalismo; relaciones internacionales.

Fecha de recibido: 21/10/2018 Fecha de evaluación: 5/03/2019 Fecha de aprobado: 28/03/2019

* Articulo preparado a partir del proyecto de tesis doctoral del autor: "La economía política internacional en la relación corrupción - desempeño económico: un estudio comparado de casos de América del Sur y el Este Asiático (1990-2013)". Programa de Doctorado en Estudios Americanos con especialidad en Estudios Internacionales. Instituto de Estudios Avanzados de la Universidad de Santiago de Chile.

a Candidato a doctor en Estudios Americanos, especialidad en Estudios Internacionales de la Universidad de Santiago de Chile (Usach) y magister en Estudios Internacionales de la misma universidad. Es becario Conicyt Doctorado Nacional 2017 (COD 21170139).

Correo electrónico: diego.jimenez@usach.cl.

ORCID: orcid.org/0000-0002-7408-1398 
Cómo citar: Jiménez Cabrera, D. I. (2019). Corrupción, integración y regionalismo en las relaciones internacionales: una discusión de los sustentos de su necesidad causal. Revista Relaciones Internacionales, Estrategia Y Seguridad, 14(2). pp. 11-32.

DOI: https://doi.org/10.18359/ries.3714

\title{
Corruption, Integration and Regionalism in International Relations: A Discussion of the Foundations of their Causal Need
}

\begin{abstract}
There is little doubt that corruption is the final node of a long chain of entities that are causally linked and, at the same time, is a phenomenon that can severely disrupt regional integration and regionalization processes. This belief - deeply rooted in the scientific community that studies this phenomenon- has resulted in the publication of numerous studies that do not combine research methods or epistemological approaches to elucidate robust causal models. Therefore, this review paper intends to discuss the foundations of the causal need underlying international relations studies of corruption in relation to integration and regionalism. The main conclusion suggests that greater methodological pluralism and epistemological eclecticism are required to explain causal relationships and broaden causality analysis, based on concepts that have observable and unobservable units.
\end{abstract}

Keywords: Causality; corruption; integration; regionalism; international relations.

\section{Corrupção, integração e regionalismo nas relações internacionais: uma discussão sobre o que mantém suas necessidades causais}

Resumo: Existem poucas dúvidas de que a corrupção seja o último elo de uma longa cadeia de entidades causalmente vinculadas e, ao mesmo tempo, um fenômeno que possa perturbar de forma severa os processos de integração regional, assim como de regionalização. Essa ideia, profundamente enraizada na comunidade científica que estuda esse fenômeno, levou à publicação de numerosos estudos que não combinam métodos de pesquisa ou abordagens epistemológicas que elucidem modelos causais robustos. Assim, este artigo de revisão pretende discutir os fundamentos da necessidade causal subjacente aos estudos em relações internacionais da corrupção em sua relação com a integração e o regionalismo. Como conclusão principal, destaca-se a necessidade de um ecletismo metodológico e epistemológico que permita explicar as relações causais como uma possibilidade de ampliar a análise de causalidade, com base em conceitos que possuam unidades observáveis e não observáveis a partir de um pluralismo metodológico maior.

Palavras-chave: causalidade; corrupção; integração; regionalismo; relações internacionais. 


\section{Introducción}

El análisis causal ha sido por más de cuatro décadas la base para los estudios de la corrupción; comprendido como un fenómeno propio de la ciencia política en general, y de las relaciones internacionales en particular. Empero, las discusiones y reflexiones epistemológicas acerca de la necesidad causal que sustentan los estudios sobre la materia son más bien escasos, y se concentran de forma casi exclusiva en construir modelos centrados en las perturbaciones que la corrupción genera en sus niveles de crecimiento económico, tanto nacional como per cápita sobre el principio del ceteris paribus (Cooper, Krieckhaus y Lusztig, 2006; Dutt, 2009; Leitner y Stehrer, 2013; Rose-Ackerman, 1978; Sandholtz y Gray, 2003; Subasat y Bellos, 2013). Otros tantos se concentran en analizar el problema a partir del "modelo del actor racional", suponiendo que todos los actores involucrados -ante un sistema de pesos y contrapesos que genere los incentivos adecuados (Hodgson y
Jiang, 2008)- utilizan la función pública para beneficio de agentes privados específicos con la finalidad de maximizar su riqueza de una forma que el respeto a la ley no permitiría (Rose-Ackerman, 1978). Son aún más escasos aquellos que discuten la vinculación corrupción-integración.

Por otra parte, la corrupción ha recibido un amplio nivel de discusión como un problema que se produce, inicialmente, a nivel nacional, y que puede perturbar las relaciones con otros países en lo político y lo económico. Esto último suele ocurrir cuando los Estados no son capaces de sostener en el tiempo el imperio de la ley, en las condiciones necesarias para preservar mercados abiertos a los flujos comerciales y de inversión globales que, a la vez, sean transparentes y equilibrados (Cooper, Krieckhaus y Lusztig, 2006, pp.121-124; Hodgson y Jiang, 2008; Rose-Ackerman, 2001; Sandholtz y Gray, 2003; Sandholtz y Koetzle, 2000; Zucker Boswell, 1996). Esto lleva a que la dimensión internacional del problema reciba un análisis con- 
siderablemente menor (Rose-Ackerman, 2001, pp. 243-270; Sandholtz y Gray, 2003; Sandholtz y Koetzle, 2000), y a que se focalice principalmente en la política comparada y los análisis de grandes muestras, que son revisadas y se discuten a partir de modelos estadísticos probabilísticos o econométricos (Collins, Uhlenbruck y Rodriguez, 2009; Cooper, Krieckhaus y Lusztig, 2006; Dutt, 2009; Leitner y Stehrer, 2013; Sandholtz y Gray, 2003; Sandholtz y Koetzle, 2000). Si bien es cierto que esto no implica la inexistencia de estudios cualitativos sobre los efectos de la corrupción en el Estado y el mercado (Cintra et al, 2018; Nye, 1967; Hodgson y Jiang, 2008; Ping, 2009), sí se puede afirmar que estos últimos poseen una presencia bastante menor en la discusión académica de la que tienen los estudios cuantitativos que examinan relaciones causales. De este modo, las investigaciones cuantitativas se centran en la construcción de inferencias causales, descripciones causales o análisis causal de grandes muestras. De estas tres categorías, la última consiste en la ejecución de las dos primeras etapas de modo simultáneo dentro de las investigaciones causales cuantitativas. En esta medida, el análisis causal de pequeñas muestras es el área clave a desarrollar en los estudios cualitativos (King, Keohane y Verba, 1994).

La integración, por otra parte, se entiende como un proceso que, de forma inevitable, lleva a un desbordamiento político de unidades nacionales para conformar una agenda e identidad supranacionales (Haas, 1958). Si bien es cierto que al discernir entre los procesos de integración social, política y económica, el mayor grado de integración radica en el nivel político (Nye, 1965, 1968; Puchala, 1970); también lo es que en el nivel económico los efectos de la corrupción son más visibles, especialmente en los casos de las economías más abiertas, o de las más dependientes del comercio internacional para lograr objetivos relacionados con el crecimiento económico (Charoensukmongkol y Sexton, 2011), que actualmente se da en un proceso de regionalización antes que de integración en el sentido europeo del término. En efecto, si la corrupción es acotada, generalmente afecta a sectores específicos de la economía de un país o conjunto de ellos, mientras que si es generalizada, podría afectar el proceso de integración (o regionalización, según el caso) total de un conjunto de países, dependiendo del contexto en el cual se desarrolla el proceso de integración y la naturaleza del mismo.

Teniendo en cuenta lo anterior, en el presente artículo me propongo analizar críticamente la naturaleza causal de los estudios de la corrupción en el ámbito de las relaciones internacionales. En la siguiente sección se revisarán los conceptos de causalidad y necesidad causal desde las relaciones internacionales. Después, se discutirán los 
conceptos de corrupción, integración regional y regionalismo ${ }^{1}$, y se pondrá en perspectiva el sustrato teórico Correspondiente a dichos procesos. Una vez culminada esta sección, en la siguiente se examinarán los estudios de corrupción -en relación a la integración y el regionalismo-, orientados hacia una perspectiva bi o multivariada, centrada en la correlación antes que en la causalidad. Finalmente, se presentarán las conclusiones.

\section{Causalidad en las relaciones internacionales: de las ideas y la necesidad causal}

Según David Hume (1711-1776), la causalidad se puede definir como una relación entre ideas, conceptos u objetos en un sentido y dirección determinados, que constituye el fundamento de la generación de conocimiento válido (2010). Respecto a este enunciado, postula dos percepciones: las ideas y las impresiones. Mientras que las primeras son imágenes iniciales que emergen del pensamiento y la razón, las segundas son visiones derivadas de la pasión, las sensaciones y las emociones, cuya primera aparición se da en el interior del alma humana (Hume, 2010, p.1).

Por otra parte, las percepciones, que incluyen las ideas y las impresiones, pueden ser clasificadas en simples o complejas, según la cantidad de cualidades que se destacan de un concepto, y a partir de cuyo enlace se define una entidad que se puede diferenciar de otra (Hume, 2010, p.1). Así mismo, Hume señala que las impresiones preceden a las ideas -y no al revés-, en una relación que él llama "conjunción constante" (Hume, 2010, p.4). Al sumarse a dicha "conjunción" la memoria, como componente que preserva la posición y temporalidad de las ideas, se da forma a la relación causa-efecto, cuyo sustento fundamental es el principio de regularidad, en el sentido de que dicha relación se debe verificar constantemente en el tiempo y en numerosos casos para que pueda considerarse como válida.

Entre los conceptos enunciados por Hume, la causalidad es el más relevante, en cuanto es la relación por la cual las ideas adquieren un mayor grado de conexión. Es a partir de una idea que otras pueden emerger para formar entramados complejos, sobre la base de la existencia de un objeto o acción determinados. Este era el tipo de relación más relevante para Hume, hasta el punto de señalar que relaciones tales como la localización espacial-temporal o la identidad solo se emplean en la medida que afectan o son alteradas por la causalidad (Hume, 2010, p.42).

1 El regionalismo, en su acepción clásica del siglo xIX, consiste en un proceso político en el cual el poder global tiende a fragmentarse en regiones o bloques. No obstante, en la actualidad se comprende como un proceso más poroso y polifacético, que excede al ámbito político e incorpora al efecto dimensiones económicas y culturales en las relaciones entre actores que ejercen algún grado de acción internacional. En este sentido, una región se comprende como un mundo de múltiples aristas y dimensiones antes que como una unidad geográfica (Acharya, 2014, pp. 649-652). 
Este es un sentido especialmente relevante, en cuanto la causalidad permitiría, para Hume, rastrear la composición y la existencia de objetos o ideas teóricamente relacionados más allá de nuestros sentidos. Para que esto sea posible, la relación señalada debe cumplir tres condiciones: contigüidad, sucesión y conexión necesaria (Hume, 2010, pp.42-48). Si estas condiciones se cumplen, se sostiene que los objetos comienzan su existencia al estar vinculados causalmente (Hume, 2010, pp.45-47). De no estarlo, entonces solo existen en la imaginación.

La noción de relación causal es comentada por Thomas Kuhn, quien declara que es una creencia persistente entre las comunidades científicas que los experimentos naturales (formulación de diseños y experimentos bajo una relación causal) estrechan la relación entre la teoría y la naturaleza mediante el estudio empírico constante (Kuhn, 1971, pp.51-67). En consecuencia, a partir de los experimentos se generan un conjunto de saberes, leyes, teoremas y principios, a través de los cuales se adquiere un conocimiento altamente detallado y representativo de un aspecto de la naturaleza -o un conjunto acota- do de facetas-, y que se convierten en la forma convencional de trabajo de una comunidad científica o en la lógica de la ciencia normal (Kuhn, 1971, pp.68-90). Por ende, lo que se aleja de la sociedad pertenece a la naturaleza, a lo no humano (Latour, 2007).

En el ámbito de las relaciones internacionales, en la actualidad se debate la idea de que lo examinado a partir de un esquema de relación causal semejante al de las ciencias exactas se circunscribe a la explicación de fenómenos, mientras que los estudios no causales buscan comprenderlos solamente. En este sentido, subyacen diferencias ontológicas insalvables entre lo causal y lo no causal, "aquello que se explica y lo que se comprende" (Hollis y Smith, 1991). Una de las posiciones más eclécticas ${ }^{2}$ en la materia la representa Alexander Wendt, quien postula que las teorías constitutivas -indagan en la composición de actores y fenómenos desde su contexto y constitución- (Wendt, 1998) y las teorías causales explican (indagan en el análisis causal), a la vez que permiten comprender (explorar posibilidades de interpretación del problema) los fenómenos propios de las relaciones

2 Según la Real Academia Española de la Lengua (RAE), el término "eclecticismo" posee las siguientes definiciones: 1) m. Adopción, en el juzgar u obrar, de una postura intermedia entre doctrinas o actitudes diversas; 2) m. Combinación de elementos de diversos estilos, ideas o posibilidades. Por ejemplo: eclecticismo arquitectónico; 3) m. Fil. Escuela filosófica que procura conciliar las doctrinas que parecen mejores o más verosímiles, aunque procedan de diversos sistemas. A la luz de las definiciones provistas por la RAE, los autores de la escuela inglesa, en diferentes grados, se inclinan más por la primera, dado que el eclecticismo metodológico se entiende más como una postura o propuesta intermedia entre diversas posibilidades teóricas o metodológicas, y no haciendo referencia a una escuela filosófica, por cuanto las diferencias ontológicas, e incluso epistemológicas, no son consideradas conciliables ni conjugables (Kurki, 2008; Jackson, 2011), tal como se expone posteriormente. 
internacionales, ya que la descripción es considerada esencial para dilucidar la naturaleza de las relaciones entre agentes y su conocimiento es condición sine qua non para establecer una posible relación causal a nivel analítico. En esta medida, se puede afirmar que la necesidad causal no es la única que conduce a la realización de la ciencia ni a la obtención de conocimiento (Wendt, 1998, p.117).

Empero, la crítica formulada a la visión de la necesidad causal Ilamada "Humeniana" (Kurki, 2008) proviene de la escuela inglesa de las relaciones internacionales. Los humenianos ${ }^{3}$ han presentado, de acuerdo con Kurki (2008), un concepto de relación causal estrecho y ontológicamente plano. Para proponer una vía alternativa a la causalidad humeniana, la autora postula la idea del Estado como una unidad que ontológicamente tiene significado y está compuesto de relaciones sociales, normas, valores e ideas que son entes "no observables", en un sentido estadístico, y, por ende, han sido despreciados de los análisis de sistemas cerrados clásicos del positivismo y el neopositivismo (Kurki, 2008, pp.289-310).

Una propuesta para superar este problema consiste en volver a la noción de causalidad aristotélica, aunque con un fuerte énfasis en tres aspectos esenciales para las relaciones internacionales: 1) la formulación de un contexto histórico sólido, en el sentido de que capture la complejidad de un sistema basado en entidades cuyos componentes son "no observables"; 2) superar la tensión teórica entre causalistas y constitutivistas, a partir del análisis multicausal y una perspectiva interdisciplinaria, dado que las relaciones internacionales no se apartan de la ontología de las ciencias sociales, aunque sus cultores y académicos la perciban como una disciplina intrínsecamente "dividida"; 3) aceptar la diversidad epistemológica en cuanto característica distintiva de las relaciones internacionales como disciplina (Jackson, 2011; Kurki, 2008). Respecto al tercer punto, cabe señalar que aunque la diversidad epistemológica caracteriza a las relaciones internacionales como una disciplina que ha estado dividida por un largo tiempo (Kurki, 2008), la posibilidad de conversar y deliberar entre distintas perspectivas para comprender, formular y construir ciencia puede realizarse siempre y cuando no se mezclen preceptos ontológicos, filosóficos y epistemológicos que son abiertamente contradictorios entre sí (Jackson, 2011, pp. 207-212). En este sentido, el pluralismo metodológico no implica un relativismo metodológico, sino que está dado por la capacidad de hacer dialogar vertientes metodológicas con sus propias lógicas internas y procedimientos para crear conocimiento a través de un enfoque cuya "traducción" sea factible, teniendo en cuenta las diferencias de lógica interna y validez del

3 Se consideran "humenianos", en las ciencias sociales en general, y en las relaciones internacionales en particular, aquellos que conciben la causalidad como la postuló David Hume (desde la perspectiva de la regularidad). 
conocimiento (Jackson, 2011, pp.207212).

Por otra parte, el holismo metodológico que postula James Robinson indica que debe existir una distinción clara y exhaustiva entre los elementos que componen una relación causal, sus propiedades y principios bajo la lógica de la "causa eficiente" de Aristóteles, reinterpretada por los neoaristotélicos sobre el principio del pluralismo metodológico (Robinson, 2011, p.35). En efecto, las relaciones causales pueden examinarse a nivel humano, material, institucional e ideacional, es decir, los análisis bajo la lógica de la causalidad generativa -altamente específica de las causas, y que supera los criterios de condición necesaria y suficiente- podrían aplicarse tanto al mundo social como al natural (Robinson, 2011, p.35). Para que esto último sea posible, resulta imprescindible considerar los aspectos constitutivos y la condicionalidad de relaciones individuales que son susceptibles de ser encadenados en secuencias espaciotemporales conducentes a resultados válidos, enfatizando en el tipo de condición que fundamente la relación causal entre determinadas entidades y las hipótesis a partir de las cuales dichas relaciones se estudian, entienden y explican (Mahoney, Kimball y Koivu, 2009).

\section{La corrupción, la integración regional y el regionalismo como objetos de estudio de las relaciones internacionales}

Uno de los temas centrales de la corrupción como objeto de estudio de las relaciones internacionales consiste en el debilitamiento que esta produce en el vínculo entre la seguridad económica y la estabilidad institucional de los Estados y los bloques regionales. En otras palabras, debilita el conjunto de reglas del juego comunes y vinculantes para los Estados miembros que facilitan el enlace de los mercados internacionales (Robertson, Gilley y Crittenden, 2008; Orozco, Rodríguez y Castro, 2011, pp.264-265), en una secuencia evolutiva cimentada en las siguientes etapas: unión aduanera, zona de libre comercio, mercado común y unión económica.

Una primera definición de corrupción consiste en "el mal uso de la función pública para beneficio privado" (Sandholtz y Grey, 2003, p.761). A través de esta, se puede observar una visión utilitarista basada en el individualismo metodológico (Hodgson y Jiang, 2013, pp.61-62). Esta definición resulta incompleta por dos motivos: 1) se presume al agente económico del sector público en una relación pasiva con el privado y orientada a la ganancia personal ilícita; 2) a partir de la definición precedente se soslayan de plano los componentes institucionales de la corrupción (sistema de pesos y contrapesos, sistema de remuneraciones, marco regulatorio y barreras comerciales impuestas desde el Estado, inter alia).

Como consecuencia del fenómeno descrito anteriormente se ha construido un proceso de conceptualización de la corrupción como un fenómeno 
organizacional. En efecto, Hodgson y Jiang (2008) señalan que, desde una perspectiva neoinstitucionalista, la producción de bienes privados contiene un conjunto de derechos que se validan socialmente (Hodgson y Jiang, 2008, p.63). Así, los sectores público y privado están entrelazados y son inseparables, es decir, en ambos sectores existen agentes activos y pasivos en el ámbito de la corrupción (Hodgson y Jiang, 2008, p.64). Una definición de la corrupción organizacional es la que Anand, Ashforth y Joshi (2004, en Zyglidopoulos, Fleming y Rothenberg, 2009) proporcionan, al señalar que la corrupción se define como "el mal uso de una posición organizacional o autoridad para la ganancia personal $\mathrm{u}$ organizacional (o de una subunidad), donde el mal uso se refiere a una disrupción de las normas societales aceptadas" (Zyglidopoulos, Fleming y Rothenberg, 2009, p.66).

Desde el punto de vista organizacional, las instituciones del sector público son espacios potencialmente susceptibles de padecer la corrupción. Empero, cabe señalar que no solo estas son afectadas, sino que influye también en el sector privado, por lo que los agentes económicos de las dos áreas pueden ejercer la corrupción, o bien ser víctimas de ella (Hodgson y Jiang, 2008, pp.57-64). En consecuencia, atribuir la corrupción solo a agentes económicos del sector público, especialmente desde el punto de vista del ejercicio de acciones corruptas, además de ser un error conceptual profundamente arraigado en la academia, debilita la capacidad explicativa de los estudios producidos hasta la actualidad sobre la materia y deja fuera de cualquier consideración al sector privado, a las empresas del sector público y a países con Estados pequeños, entre otros agentes de alta relevancia dentro de esta línea de investigación (Hodgson y Jiang, 2008, pp.57-75).

Otro aspecto a considerar respecto al error conceptual ya citado es que la corrupción depende fuertemente, en diversos casos, de los lazos personales entre los agentes públicos y privados, sus valores compartidos y la prevalencia de instituciones informales dentro del Estado (Ping, 2009), aspecto que quedaría descartado como objeto de análisis si la corrupción solamente tuviera espacio dentro del sector público.

Entonces, la discusión sobre la materia genera la necesidad de responder una pregunta clave: ¿de qué modo influye la corrupción, comprendida en su sentido organizacional, sobre el crecimiento económico de un Estado? Una primera aproximación la proporcionan Cooper, Krieckhaus y Lusztig (2006), al indicar la existencia de dos grandes posiciones sobre el vínculo entre corrupción y crecimiento económico. Estas dos posturas son las que se examinan a continuación.

La visión proveniente de la corriente neoinstitucionalista arguye que la corrupción produce una fuerte desviación del crecimiento de la economía desde el punto de vista de la oferta, tanto desde la lógica de un impuesto 
a la producción como desde las distorsiones que genera en el mercado (Cooper, Krieckhaus y Lusztig, 2006, pp.122-123). Este fenómeno genera como efecto altos costos de oportunidad para invertir los recursos disipados - debido al surgimiento de un mercado de los favores y la influencia políticaen la actividad productiva y, por ende, en el crecimiento económico (Cooper, Krieckhaus y Lusztig, 2006, p.123). En efecto, la disminución de la inversión se explica por los altos costos de transacción que la corrupción genera, lo que es una muestra del debilitamiento de la confianza en las instituciones políticamente organizadas, si nos remitimos al concepto de corrupción organizacional enunciado anteriormente.

La segunda perspectiva del problema, defendida por los monetaristas, consiste en que la corrupción es económicamente fértil para la actividad productiva, en cuanto obliga a las firmas pequeñas a ser más eficientes para poder competir en un mercado condicionado por las influencias y favores políticos. En efecto, las empresas menos eficientes son obligadas a salir de ese mercado negro (Cooper, Krieckhaus y Lusztig, 2006, p.123), lo que implica que aquellas más grandes provean una base más amplia de ingresos fiscales vía impuestos y, por extensión, de gasto público; dicha base resulta generada a partir de recursos reinvertidos por el Estado (p.123). Esto lleva a considerar que la corrupción, para quienes adhieren a la escuela ya señalada, es beneficiosa a pesar de distorsionar el mercado y el crecimiento económico, pues son externalidades negativas consideradas menores respecto a aquellas que puede causar un Estado con altos niveles de transparencia, pero que tiene una burocracia demasiado grande (pp.123-124). En efecto, los monetaristas parten de la premisa de que es el tamaño del Estado per se el mayor distorsionador de la economía y, a la vez, la principal amenaza al crecimiento económico. Así, las distorsiones provocadas por la corrupción son más tolerables que una mayor intervención del Estado en la conducción de la economía, e incluso puede atraer, como en América Latina, inversión extranjera directa de corporaciones multinacionales que podrían ser generadoras de corrupción antes que víctimas de prácticas corruptas (Subasat y Bellos, 2013, pp.153-155).

Si consideramos la visión institucionalista como de interés para nuestro estudio, entonces, cabe preguntarse: ¿cómo afecta la corrupción las relaciones entre dos o más Estados? Una primera aproximación la proporcionan Sandholtz y Grey (2003), quienes identifican un conjunto de factores internacionales que producen efectos a nivel interno de los países y, en consecuencia, vinculan los mercados externos con los internos. Entre los principales factores, los autores ya citados destacan los siguientes: las crisis económicas internacionales; la apertura de los mercados externos; los cambios relativos de los precios en los mercados internacionales; las redes transnacionales de activistas; las comunidades epistémicas internacionales, y las or- 
ganizaciones internacionales y supranacionales (Sandholtz y Grey, 2003, pp.763-764). Para efectos de la presente discusión, nos interesan dos de estos factores: la apertura de los mercados externos y las organizaciones internacionales y supranacionales.

En relación al primer factor (la apertura de los mercados externos), la corrupción constituye un factor de la mayor preocupación. La instauración de reformas sistemáticas orientadas a abrir de forma progresiva los mercados con el fin de maximizar el crecimiento a través de la globalización de cadenas de valor, comercio e inversiones han tenido como principal corolario la idea de que la apertura de los mercados globaliza los beneficios también (Michael, 2012) y, con ello, favorecen el crecimiento y el desempeño económico. No obstante, algunos autores sostienen, con respecto a esta idea, que la relación no solo es débil, sino que es viable el control de la corrupción con restricciones al comercio internacional (Torrez, 2002, pp.399-401). Un segundo elemento relevante consiste en que, más allá de que los mercados estén abiertos o no, lo importante es conocer la cultura y la lógica con la cual se construye la confianza entre los funcionarios del sector público y los agentes económicos del sector privado (Li y Wu, 2010). Si el uso de redes de actores y contactos clave es vital para la generación de relaciones económicas de cualquier tipo (confianza generalizada), entonces, los efectos de la corrupción se vuelven focalizados y, por ende, no afectan mayormente el crecimiento ni el desarrollo de la integración económica, como en el caso de China y otros países del Este Asiático (Li y Wu, 2010, pp.147-150).

En relación con las instituciones supranacionales, cabe señalar que estas últimas reflejan el estado de la discusión acerca de la corrupción y la integración. Si bien es cierto que los esfuerzos estatales y no estatales por generar normas homólogas para castigar las conductas y prácticas corruptas han sido numerosos, especialmente desde inicios de la década del 90 del siglo XX, también existen déficits relevantes, tales como la ausencia de un concepto de corrupción que permita la configuración de normas comunes más efectivas para su control a partir de la Convención de Naciones Unidas para el Control de la Corrupción (Argandoña, 2007, p.488), tanto desde la perspectiva de la economía política internacional como desde la seguridad internacional (Sanahuja, 2012). Esta última tuvo la intención inicial de que los signatarios desarrollaran toda su normativa como parte del espíritu general de la Convención, no obstante, las diferencias entre las prácticas y su asociación al concepto de corrupción llevan a que uno de los problemas esenciales en la arquitectura económica internacional sea la definición de la misma antes que la simple enumeración de sus prácticas constitutivas (Li y Yu, 2014, pp.153-154).

Este problema se ha vuelto aún más complejo con la evolución de las re- 
laciones económicas internacionales basadas en el comercio internacional y los flujos de inversión. Si bien es cierto que estas relaciones han tendido, de un modo u otro, a ser formalizadas jurídicamente mediante tratados de libre comercio o tratados regionales de Comercio, cabe señalar que, contrario a lo que se ha señalado constantemente, no son los causantes de incrementar los flujos de recursos ni los retornos que se obtienen de los mismos, sino que solo confirman el desarrollo de relaciones que, en el ámbito de la economía política internacional, se han mantenido lo suficientemente estables para dar paso a la conformación de entidades que faciliten la coordinación política y doten de mayor estabilidad política y certeza jurídica a dichos flujos (Baier, Bergstrand, Egger y McLaughlin, 2008, pp.492-493). Esto último aplica con gran fuerza en América del Sur, salvo en algunos aspectos del caso de Brasil (Dingemans y Ross, 2012).

Por otra parte, uno de los mayores desafíos que enfrenta América Latina en materia de integración regional es el combate a la corrupción, que representa un factor clave en el deterioro de las instituciones nacionales (Apaza, 2009; Fredriksson y Vollebergh, 2009; Hodgson y Jiang, 2008; Leitner y Stehrer, 2013; Orozco, Rodríguez y Castro, 2010; Pandey, 2010; Valenzuela, 2011), así como de las internacionales, por dos motivos: 1) como fenómeno organizacional, la corrupción debilita la calidad institucional de los Estados, independientemente de si el agente activo (corruptor) es del sector público o del privado; 2) al debilitarse la confianza entre los agentes económicos de los dos principales sectores de la economía (público y privado), la integración de un país con respecto a un bloque regional o subregional puede verse debilitada en razón de su incapacidad para garantizar el desenvolvimiento del mercado bajo un conjunto de reglas transparentes y competitivas.

\section{Desde la integración regional al regionalismo: perspectivas teóricas}

La integración es uno de los pocos procesos estudiados en las relaciones internacionales cuya teoría ha sido estudiada de forma contemporánea al objeto de estudio de su mayor interés: la integración europea del siglo XX. Si bien es cierto que este ámbito de investigación cuenta con una producción académica destacable desde los trabajos de David Mitrany en los años 30 con la perspectiva funcionalista, $y$ a partir de los 50 con el enfoque neofuncionalista, los esfuerzos por diseñar una teoría de la integración fracasaron en su intento original: explicar por qué ocurrió la integración europea (Malamud y Schmitter, 2006, pp. 3-8).

Más allá de los enfoques referidos, el problema central radica en entender que la integración regional es, como fue concebido por Joseph S. Nye (1965; 1968), ante todo, un proceso de interdependencia que puede variar según si se establece o no como supuesto la división entre alta y baja política (Nye, 1965, pp.870-873). Así mismo, la continuidad institucio- 
nal de la integración es, más bien, un supuesto basado en el nivel de centralización del poder político a nivel nacional, que luego es conducido al nivel de la decisión supranacional (Nye, 1965, p.873).

Si se comprende que el proceso de integración regional es un proceso de armonización de fines, recursos, instituciones y decisiones, a nivel supranacional, entre unidades geográficamente vecinas (Nye, 1968), la diferenciación entre la integración política, la integración económica y la integración social es una implicación necesaria. Entre este conjunto de diferencias esenciales, la más importante de todas radica en que, mientras la integración política apunta a la formación de una comunidad para perseguir intereses comunes (Nye, 1968, p.865), la integración económica apunta a la disminución de la discriminación en las transacciones económicas entre los actores en los siguientes niveles: área de libre comercio (sin aranceles ni cuotas), unión aduanera (arancel externo común), mercado común (libre circulación de factores productivos), unión económica (armonización de políticas económicas) e integración económica total (unificación de políticas públicas e instituciones políticas) (Nye, 1968, pp.860-862). En consecuencia, la integración social apunta a constituir una comunidad de seguridad basada en una identidad y una comunicación en común, que generen un sentido de obligación entre sus miembros (Nye, 1968, pp.862-864).
De este modo, todas las categorías de integración de Nye apuntarían, finalmente, a la integración política como el máximo nivel de integración posible, dado que la formación de una comunidad exigiría un proceso anterior de amalgamación política (Puchala, 1970, pp.753-763) a partir del análisis de transacciones entre las unidades nacionales. En este sentido, los cambios de sistema de pensamiento dentro del ámbito de las relaciones internacionales ha replanteado la necesidad de establecer sistemas de análisis de causalidad en el contexto de la revolución conductista (Banks, 1969, pp.335-339; Nye, 1968).

Empero, estos modelos, que eran aplicables durante los años 60, 70 y 80, comenzaron a fracturarse. La evolución de las relaciones internacionales como disciplina, especialmente en el caso de la escuela inglesa, han dado un vuelco considerable al plantear la necesidad de comprender las relaciones internacionales como "globales" disciplinariamente (Acharya, 2014). Con respecto a este punto, el autor enfatiza en la idea central de que las relaciones internacionales de enfoque eurocéntrico, así como de pensamiento occidental, tienden a comprender todos los mundos posibles en términos "europeístas" u "occidentales". En efecto, la crítica principal formulada por Acharya (2014) postula que es a través de la historia que podemos comprender que las regiones, antes que entidades burocratizadas -por medio de organizaciones internacionales-, son "mundos" diferentes dentro de 
un mundo regionalizado, entendido como entidades dentro de las cuales hay unidades (estatales o no) que organizan por sí mismas sus propios espacios políticos y económicos, así como su relación con las demás unidades, y que después determinan el orden global (pp.649-652).

Entonces, dentro del ámbito de la corrupción y la integración regional, los flujos que se desarrollan dentro de estos espacios regionales tienen particularidades que superan considerablemente los principios de regularidad y de causalidad "humeniana". Estos planteamientos han sido recogidos por la escuela inglesa; específicamente, dichos flujos han comenzado a ser investigados a partir del concepto de sociedad internacional de Bull, aunque con importantes acotaciones de los niveles de análisis, con privilegio de los niveles regionales y subglobales (Pourchot y Stivachtis, 2014, pp.68-69). Así mismo, estos nuevos niveles de análisis han instado a algunos autores a reenfocar los esfuerzos en dos áreas de interés de las relaciones internacionales: 1) comprender los aspectos que constituyen las regiones como espacios en los cuales se dan flujos económicos y políticos no burocratizados; 2) que la intensidad de estos flujos condicionan en diferentes grados el control de la corrupción, así como sus impactos en el desempeño económico de Estados que conforman sociedades internacionales de escala regional. No obstante, estos últimos planteamientos requieren de estudios de caso para verificar la existencia de "poderes causales" (Kurki,
2008) que faciliten la observación del entramado causal complejo que existe dentro de una relación del tipo , así como de los elementos que componen dicha red. En este aspecto, la relación entre corrupción y desempeño económico está lejos de ser dilucidada, aunque los estudios de aspectos comunes entre los conceptos de integración regional y de regionalismo podrían proporcionar puentes para comparar casos que nos acerquen a mejores explicaciones para estos problemas, desde las relaciones internacionales en general, y la economía política internacional en particular.

\section{Corrupción, integración y regionalismo: comprensión de sus mecanismos causales a partir de las relaciones internacionales}

La corrupción y la integración regional han sido considerados como partes de un sistema causal. En el caso de la corrupción, se comprende como el principal obstáculo para el crecimiento económico en el nivel nacional, mientras que para la dimensión internacional la mayor preocupación se deposita en que los acuerdos alcanzados entre las partes favorezcan el respeto a la ley, la protección recíproca de inversiones y la gobernanza global, entendida esta última bajo un estricto individualismo metodológico, esto es, como la sumatoria de niveles de gobernanza de cada Estado participante en la sociedad internacional. Por otra parte, el segundo concepto (la integración regional) es un proceso que tiende a unificar estructuras en los Estados con la lógica 
de un sistema interdependiente, en el cual los problemas, así como los fenómenos que horadan la confianza entre las unidades nacionales, también pasan a ser compartidos.

Si se extrapolan las afirmaciones antes expuestas en el ámbito de las relaciones internacionales, esto es, a nivel de relaciones relevantes para la sociedad internacional, en la cual al menos uno de los actores ejerce su acción política a nivel supranacional (Ortiz, 2011), entonces, el individualismo metodológico se da cuando se comprenden las variables en un esquema de separación perfecta entre dependientes e independientes, que pueden tener diferencias ontológicas sustantivas que, finalmente, no las hagan evaluables bajo una lógica causal (Robinson, 2011, pp.12-13).

Lo anterior es especialmente cierto para los estudios que incluyen grandes muestras. En este sentido, el individualismo metodológico lleva a suprimir del análisis científico aquellas características o factores que hagan únicas a cada una de las entidades incluidas en el estudio, para homologarlas descartando las características morales de sus sociedades y comprender de qué forma la corrupción distorsiona los mercados y las acciones que emprenden las instituciones a nivel nacional (Boehm y Graff, 2009).

La supresión total de las particularidades de las entidades estudiadas no solo conduce a un problema normativo, sino metodológico. En relación a este último, es preciso realizar dos consideraciones: 1) las grandes muestras (sobre 20 casos) implican, la mayoría de las veces, la necesidad de hacer estiramientos conceptuales de tal envergadura que los conceptos pierden por completo su significado original (Landmann, 2011; Sartori, 2011); 2) a medida que se agrandan las muestras, solo se logran confirmar patrones o relaciones causales sobre aspectos de baja especificidad en relación al objeto de estudio que se desea investigar. De esta forma, son los estudios de pequeñas muestras los que permiten investigar más profundamente determinados mecanismos causales (Landmann, 2011). Adicionalmente, cabe señalar que la forma de establecer las muestras es decisiva respecto de este último punto, ya que, en el ámbito de las relaciones internacionales de enfoque comparado, la selección de los casos no se desarrolla bajo una lógica estadística, sino que, incluso, puede tener fundamentos lógicos que otorgan cierta arbitrariedad al proceso de selección de unidades explicativas pertinentes (Gerring, 2007; Landmann, 2011; Ragin, 1987).

Si bien es cierto que hay estudios que enfatizan en el uso de correlaciones de grandes muestras en rasgos generales asociados a la integración económica internacional de los actores, o su nivel de adscripción a organizaciones internacionales (Sandholtz y Grey, 2003, p.779-786; Sandholtz y Koetzle, 2000), estos no implican lógicamente causalidad, dado que la explicación y la predicción no constituyen elemen- 
tos suficientes para dilucidarla, por no ser capaces por sí mismos de develar mecanismos causales robustos (Robinson, 2011). En efecto, se puede afirmar que la mayoría de los estudios de corrupción, bien sea desde la ciencia política o desde las relaciones internacionales, se focalizan en el análisis estadístico cuantitativo -ya sean formulados bajo la lógica de la inferencia causal o de la inferencia descriptiva (Wendt, 1998)-, antes que en incorporar consideraciones históricas o de otro orden por ser concebidos como precientíficos (Wendt, 1998). De este modo, los procesos de integración poseen indicadores más atingentes al concepto por centrarse en el análisis de transacciones entre las unidades nacionales. Empero, la corrupción como objeto de estudio enfrenta un problema que ha sido considerado relevante para numerosos autores: al recopilarse las percepciones, tanto de expertos como de individuos, no hay forma de medir directamente la relación en que este concepto esté involucrado, por lo que no se puede evaluar consistentemente (Lin y Yu, 2014; Sandholtz y Grey, 2003; Sandholtz y Koetzle, 2000; Torrez, 2002). Esto llevaría a que solo fuera posible la descripción causal a nivel estadístico de los datos, y no el análisis causal.

De esta forma, salvo los avances de la escuela neoinstitucionalista de la economía política (Hodgson y Jiang, 2008; Boehm y Graff, 2009), y del realismo crítico en las relaciones internacionales (Robinson, 2011), se puede observar el desarrollo de una ciencia normal, centrada en construir o pulir modelos estadísticos o econométricos que miden la corrupción en torno al nivel de crecimiento y desarrollo económico de las entidades consideradas (Collins, Uhlenbruck y Rodríguez, 2009; Cooper, Krieckhaus y Lusztig, 2006; Dutt, 2009; Leitner y Stehrer, 2013; Sandholtz y Gray, 2003; Sandholtz y Koetzle, 2000). Así, el neoinstitucionalismo y el realismo crítico refuerzan sus estudios en el análisis de casos, el cual se observa una mayor propensión por los estudios cualitativos (Nye, 1967; Hodgson y Jiang, 2008; Ping, 2009), y que suelen destacar rasgos de carácter organizacional o individual sobre la corrupción. Sin embargo, dichos estudios de caso son de un solo país, lo cual debilita considerablemente el valor de sus datos, por no ser concebidos para lograr una eventual generalización de los resultados de los estudios comentados (Landmann, 2011). Además, se desarrollan a nivel de los procesos de integración cuando se examinan bloques, pues, aunque aumente la cantidad de países en el análisis, son las regiones las que constituyen casos, antes que las unidades nacionales, desde la perspectiva de las relaciones internacionales. El problema de la selección de pocos casos obliga a una mayor acumulación de datos, a aumentar la cantidad de variables, o bien, a examinar los países como caso de forma complementaria al examen de las regiones solamente.

No obstante, la salida alternativa más plausible es la utilización del concepto de regionalismo. Esto último per- 
mite dar solidez a la comparación de casos, prescindiendo de un concepto de integración que tiene en su centro la conformación de instituciones políticas que han sido logradas, en gran parte por la Unión Europea, y en parte por algunos estados de América del Sur mediante el Mercosur, aunque con resultados altamente dispares.

\section{Conclusiones}

Si bien es cierto que la corrupción, la integración y el regionalismo como objetos de estudio en el ámbito de las relaciones internacionales es más bien reciente -considerado el volumen de producción académica que la examina desde la perspectiva de las relaciones internacionales y, específicamente, de la economía política internacional-, es posible observar algunos aspectos críticos en su desarrollo.

En primer lugar, en los estudios realizados se reflexiona escasamente respecto a la necesidad causal que sustenta la investigación sobre corrupción, integración regional y regionalismo. Esto lleva a que se construyan modelos estadísticos o econométricos que no indagan en necesidades de otro tipo, tales como podrían ser la necesidad histórica o la necesidad lógica, entre otras que nos permitirían conocer los entramados de relaciones causales existentes entre una o más variables independientes y una dependiente. De esta forma, los estudios señalados priorizan el empleo de grandes muestras antes que la construcción de conceptos que den cuenta de una hipotética relación causal, y menos aún la descripción de las entidades que actúan para que se produzcan o la vinculación entre ellas. En efecto, los estudios que trabajan con grandes muestras tienden a describir más la estructura y el propósito de los modelos empleados que a producir una descripción detallada de las relaciones entre las entidades, o bien, de proceder a estudiar la pertinencia de la inferencia causal. En este sentido, parece plausible el planteamiento de Alexander Wendt, en cuanto a que el entendimiento y la explicación en el campo de las relaciones internacionales no son mutuamente excluyentes, sino que son altamente complementarias. Las posiciones de Kurki y Jackson también pueden ser alternativas razonables para alcanzar dicha meta.

Un problema esencial para comprender los mecanismos causales entre corrupción e integración se encuentra en el concepto de muestra y en su selección. En este sentido, el no se selecciona por fundamentos estadísticos, sino por motivos lógicos en la mayoría de los casos, lo cual supone un alto riesgo de sesgo en la selección. Una segunda dificultad radica en que, a nivel de los procesos de integración vinculados a corrupción, se produce un análisis de casos a dos niveles: el de la región en sí misma, y el de las unidades nacionales que la componen, lo que puede determinar un análisis multinivel. Empero, una alternativa interesante para salvar este obstáculo es trabajar de forma multidisciplinaria, especialmente en el caso de la incorporación del análisis histórico, así como de un eclecticismo 
metodológico que admita usar diferentes lentes para observar un mismo problema. Esto puede satisfacer necesidades de orden histórico y lógico que el análisis estadístico por sí mismo no suple en su totalidad, a fin de construir conceptos y relaciones causales más robustas y reconstruir procesos secuenciales que permitan sustentar de forma más adecuada el planteamiento de relaciones de este tipo.

Respecto a lo anteriormente señalado, cabe mencionar que existe una proclividad altamente arraigada en la comunidad científica a los estudios cuantitativos -antes que a los cualitativos-, en los campo de la economía política internacional de la corrupción, el regionalismo y la integración regional, en los cuales se han concentrado en pulir modelos para estudiar la corrupción como variable dependiente en relación con otras variables vinculadas a la calidad del sistema político y al desempeño económico, tal como ocurre en el caso de la integración. Este tema conduce a controversias complejas que se centran en la pertinencia del modelo a utilizar, y no a discutir sus fundamentos epistemológicos ni a debatir la necesidad de los métodos mixtos o el eclecticismo metodológico para dar un nuevo impulso a los estudios sobre la materia. En este sentido, la comunidad científica ha construido una ciencia normal en torno a la corrupción y, desde la primera cuantificación de datos relativos al concepto (1995), se ha enfocado principalmente en construir modelos correlacionales o econométricos que se han corregido a través de los años. Esta tendencia a estudiar la causalidad desde la estadística inferencia también se ha mantenido en los estudios sobre la integración regional y la regionalización. En esta medida, la relación entre estos tres procesos ofrece interesantes perspectivas de desarrollo en las relaciones internacionales, si esta disciplina se abre a un grado creciente de pluralismo metodológico.

Finalmente, cabe señalar un aspecto importante sobre el pluralismo metodológico. Aunque el concepto esbozado por Jackson (2011) apunta a respetar las lógicas internas y la validez de las diferentes perspectivas metodológicas para hacer posible un diálogo desde las relaciones internacionales, no resulta menos cierto el hecho de considerar el concepto de necesidad que moviliza la producción de conocimiento en relaciones internacionales. En efecto, el concepto de necesidad causal, por ejemplo, requiere de la unificación de conceptos derivados de una necesidad lógica por sí misma, o bien, inspirarse en necesidades de otro tipo para establecer luego una relación causal. En este sentido, los métodos, entendidos como formas o instrumentos para reunir y sistematizar datos, pueden apoyar la observación de fenómenos y unidades para resaltar determinados aspectos de la relación causal de base del fenómeno que se desea estudiar. Del mismo modo, los métodos pueden robustecer la lógica interna y las conclusiones de la investigaciones en relaciones internacionales. Empero, 
estos no pueden unir las formas de concebir y sustentar la causalidad ontológica y epistemológicamente, por cuanto, al entrar en estas últimas dos dimensiones, hablamos de formas de entender el vínculo mente-mundo cuyas diferencias resultan insalvables.

\section{Referencias}

Acharya, A. (2014). Global international relations (IR) and regional worlds. International studies quarterly, 58, 647-659.

Anand, V., Ashforth, B. E. y Joshi, M. (2004). Business as usual: The acceptance and perpetuation of corruption in organizations. Academy of Management Executive, 18(2), 39-53.

Apaza, C. (2009). Measuring governance and corruption through the worldwide governance indicators: critiques, responses, and ongoing scholarly discussion. PS: Political Science and Politics, 42(1), 139143.

Argandoña, A. (2007). The United Nations Convention against corruption and its impact on international companies. Journal of Business Ethics, 74(4), 481-496.

Baier, S. L.; Bergstrand, J. H.; Egger, P., yMcLaughlin, P. A. (2008). Do economic integration agreements actually work? Issues in understanding the causes and consequences of the growth of regionalism. The World Economy, 31(4), 461-497. Recu- perado de doi: 10.1111/j.14679701.2008.01092.x

Banks, M. (1969). Systems analysis and the study of regions. International Studies Quarterly, 13(4), 335-360.

Boehm, F. y Graf, J. (2009). Corrupción y anticorrupción: una perspectiva neo-institucional. Revista de ECOnomía Institucional, 11(21), 45-72.

Charoensukmongkol, P. y Sexton, S. (2011). The effect of corruption on exports and imports in Latin America and the Caribbean. Latin American Business Review, 12(2), 83-98.

Cintra, R. F. et al. (2018). Corruption and emerging markets: Systematic review of the most cited. Research in International Business and Finance, 45, 607-619.

Collins, J.; Uhlenbruck, K. y Rodríguez, P. (2009). Why firms engage in corruption: a top perspective. Journal of Business Ethics, 87(1), 89-108.

Cooper Drury, A.; Krieckhaus, J. y Lusztig, M. (2006). Corruption, democracy and economic growth. International Political Science Review, 27(2), 121-136.

Dingemans, A. y Ross, C. (2012). Free trade agreements in Latin America since 1990: an evaluation of export diversification. Cepal Review, (108), 27- 48.

Dutt, P. (2009). Trade protection and bureaucratic corruption: An empirical investigation. The Canadian Journal of Economics, 42(1), 155183. 
Fredriksson, P. y Vollebergh, H. (2009). Corruption, federalism and policy formation in the OECD: The case of energy policy. Public Choice, 140(1 - 2), 205-221.

Gerring, J. (2007). Case study research. Principles and practices. Cambridge: Cambridge University Press.

Hodgson, G. y Jiang, S. (2008). La economía de la corrupción y la corrupción de la economía: una perspectiva institucionalista. Revista de Economía Institucional, 10(18), 55-80.

Hollis, M. y Smith, S. (1991). Explaining and understanding international relations. Oxford: Clarendon Press.

Jackson, P. T. (2011). The conduct of inquiry in international relations: philosophy of science and its implications for the study of world politics. New York: Routledge.

Haas, E. B. (1958). The uniting of Europe: political, social and economic forces, 1950-1957. Indiana: Notre Dame University Press.

Hume, D. (2010). Treatise of human nature. Book I: The understanding. United States: Jonathan Bennet.

King, G., Keohane, R. O. y Verba, S. (1994). Designing social inquiry. Scientific inference in qualitative research. Princeton: Princeton University Press.

Kuhn, T. S. (1971). La estructura de las revoluciones científicas. México D.F.: Fondo de Cultura Económica.
Kurki. M. (2008). Causation in international relations. Reclaiming causal analysis. Cambridge: Cambridge University Press.

Landmann, T. (2011). Política comparada. Una introducción a su objeto y métodos de investigación. Madrid: Alianza Editorial.

Latour, B. (2007). Nunca fuimos modernos. Ensayo de Antropología Simétrica. Buenos Aires: Siglo Veintiuno Editores.

Leitner, S. y Stehrer, R. (2013). Access to finance and funding composition during the crisis: A firm-level analysis of Latin American countries. Journal of Latin American Economics, 50(1), 1-47.

Li, S. y Wu, J. (2010). Why some countries thrive despite corruption: The role of trust in the corruption-efficiency relationship. Review of International Political Economy, 17(1), 129-154.

Lin, M. W. y Yu, C. (2014) Can corruption be measured? Comparing global versus local perceptions of corruption in east and southeast Asia. Journal of Comparative Policy Analysis: Research and Practice, 16(2), 140-157.

Mahoney, J.; Kimball, E. y Koivu, K. L. (2009). The logic of historical explanation in social sciences. Comparative Politics Studies, 42(1), 114-146.

Malamud, A. y Schmitter, P. C. (2006). La experiencia de integración europea y el potencial de integración 
del Mercosur. Desarrollo Económico, 46(181), 3-31.

Michael, B. (2012). Do customs trade facilitation programs help reduce customs-related corruption? International Journal of Public Administration, 35(2), 81-97.

Nye, J. S. (1965). Patterns and catalysts in regional integration. International Organization, 19(4), 870-884.

Nye, J. (1967). Corruption and political development: A cost-benefit analysis. The American Political Science Review, 61(2), 417-427.

Nye, J. S. (1968). Comparative regional integration: Concept and measurement. International Organization, 22(4), 855-880.

Ortiz, E. (2011). El estudio de las relaciones internacionales. Santiago de Chile: Fondo de Cultura Económica.

Orozco, G.; Rodríguez, G. y Castro, C. (2010). Proyección internacional y estabilidad regional. El caso de Brasil y el Mercosur en la política internacional. Investigación y Desarrollo, 18(2), 242-269.

Pandey, P. (2010). Service delivery and corruption in public services: How does history matter? American ECOnomic Journal: Applied Economics, 2(3), 190-204.

Ping, P. (2009). The duality of crony corruption in economic transition: An integrated framework. Journal of Business Ethics, 85(1), 41-55.

Pourchot, G. y Stivachtis, Y. A. (2014). International society and integra- tion in Central Asia. Journal of Eurasian Studies, 5, 68-76.

Puchala, D. J. (1970). International transactions and regional integration. International Organization, 24(4), 732-763.

Ragin, C. (1987). The comparative method. Moving beyond qualitative and quantitative strategies. Berkeley: University of California Press.

Real Academia Española de la Lengua. Diccionario de la Lengua Española. Trigésimo Centenario. Recuperado de https://dle.rae.es/?id=EKLBSQr

Robertson, C.; Gilley, K. M. y Crittenden, William F. (2008). Trade liberalization, corruption and software piracy. Journal of Business Ethics, 78(4), 623-634.

Robinson, J. F. (2011). What causes IR? Differentiating causal analyses in international relations. APSA Conference. Seattle. September.

Rose-Ackerman, S. (1978). Corruption: A study in political economy. New Haven: Academic Press.

Rose-Ackerman, S. (2001). La corrupción y los gobiernos. Causas, consecuencias y reforma. Madrid: Siglo Veintiuno de España Editores.

Sanahuja, A. (2012). Post-liberal regionalism in South America: the case of Unasur. EUI Working Paper 05. European University Institute (EUI). Robert Schuman Centre for Advanced Studies, Florencia.

Sandholtz, W. y Gray, M. (2003). International Integration and National 
Corruption. International Organization, 57, 761 - 800.

Sandholtz, W. y Koetzle, W. (2000) Accounting for corruption: Economic structure, democracy, and trade. International Studies Quarterly, 44(1), 31- 50.

Sartori, G. (2011). Cómo hacer ciencia política. Lógica, método y lenguaje en las ciencias sociales. Madrid: Editorial Taurus.

Subasat, T y Bellos, S. (2013). Corruption and foreign direct investment in Latin America: A panel gravity model approach. Journal of Management and Sustainability, 3(4), 151-156.

Torrez, J. (2002). The effect of openness on corruption. The Journal of International Trade \& Economic Development, 11(4), 387-403.
Valenzuela, B. (2011). Comercio y calidad institucional en el crecimiento económico del Cono Sur de América: Cuestiones y desafíos. Revista Pilquén, 13(14), 1-8.

Wendt, A. (1998). On constitution and causation in international relations. Review of International Studies, 24, 101-117.

Zucker Boswell, N. (1996). Combating Corruption: Are International Institutions Doing Their Job? American Society of International Law, 90, 98-105.

Zyglidopoulos, S.; Fleming, P. J. y Rothenberg, S. (2009). Rationalization, overcompensation and the escalation of corruption in organizations. Journal of Business Ethics, 84(1), 65-73. 